\title{
AUTO-TUNING PID CONTROLLERS IN TERMS OF RELATIVE DAMPING
}

\author{
F. Morilla*; A. González** ; N. Duro* \\ * Dpto de Informática y Automática, UNED, Avda. Senda del Rey s/n, 28040 Madrid, \\ Spain. Phone:34-91-3987156 Fax:34-91-3986697, \\ E-mail:fmorilla@dia.uned.es; nduro@dia.uned.es \\ ** Dpto de Ingeniería Mecánica y Diseño Industrial, Escuela Universitaria Politécnica, \\ C/. Sacramento 82, 11003 Cádiz, Spain. Phone:34-56-221766 Fax:34-56-224359, \\ E-mail: antonio.gonzalezlopez@uca.es
}

\begin{abstract}
In this paper a new auto-tuning method of PID controller is proposed. It combines: modelling of the closed-loop system, modelling of the process, and tuning formulas in terms of the relative damping of the transient response to setpoint changes. So that is possible a greater range of specifications in the system response and to provide formulas of estimation and tuning which could be useful in other strategies. The method has been tested in simulation and in real time with SIMULINK. The method produces the best performance with respect to other methods for process with dominant dead time, which are considered the most difficult ones to control. Copyright () 2000 IFAC
\end{abstract}

Keywords: automatic tuning, pattern recognition, PID control, relative damping.

\section{INTRODUCTION}

It is a widely known that the PID controller possesses the capacity to resist changes in the process without excessive deterioration of the control loop behaviour. Despite its robustness, in specific situations the PID controller also needs an auto-tuning strategy. Basically, two types of strategies have prevailed in commercial regulators, those based on the analysis of the transient response (pattern recognition approach) and those based on the frequency response (Aström et al., 1993; Aström, and Hägglund, 1995).

In some auto-tuning controllers the characteristics determined by the "system response analyzer" are directly used by the "control parameters tuner", as consequence the relation between the specifications and the PID parameters is normally quite involved, and heuristic and logic are required (Kraus and Myron, 1984; Seem, 1996). However, in other methods such as that proposed by Morilla (1987), the response characteristics are used to estimate the parameters of a process model, and tuning formulas are available for limited ranges of the specification.

In this paper a new auto-tuning method of PID controller is proposed, which combines: modelling of the closed-loop system, modelling of the process, and tuning formulas in terms of the relative damping of the transient response to setpoint changes. So that is possible a greater range of specifications in the system response and to provide formulas of estimation and tuning which could be useful in other strategies. In section 2 the main characteristics of the method are presented. In section 3 the tests for validation are mentioned. In section 4 some applications of the method are tested in simulation with a well-known system. Finally the paper is completed with the conclusions in section 5 .

\section{CHARACTERISTIC OF THE AUTO-TUNING METHOD}

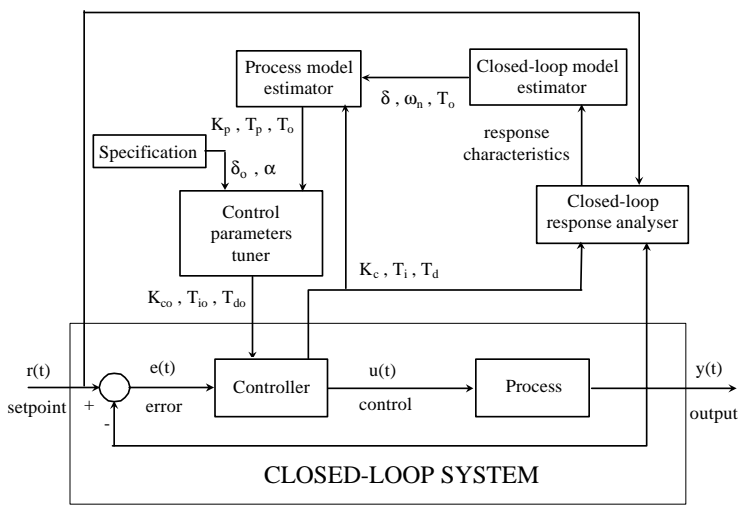

Fig. 1. Diagram of proposed auto-tuning method.

The proposed auto-tuning method allows to get different situations of transient response using a specification, the relative damping or any other temporal characteristic directly related with it, as for 
example the decay ratio or the maximum overshoot. The method involves four well-defined stages (see Figure 1): analysis of the closed-loop transient response, estimation of the closed-loop model, estimation of the process model and tuning of the control parameters.

The system response to setpoint changes is registered until there is enough information to determine its type and its characteristics. The closed-loop model estimation find the parameters $\left(\delta, \omega_{n}, T_{o}\right)$ of a continuos second order system with dead time (see Figure 2), which better approximates the characteristics of the system response. The process model estimation determines the parameters $\left(\mathrm{K}_{\mathrm{p}}, \mathrm{T}_{\mathrm{p}}\right.$, $\mathrm{T}_{\mathrm{o}}$ ) of a continuous first order model with dead time (see Figure 2) such that the transfer function of the pseudo system and that of the closed-loop model are equivalent. The control parameters $\left(\mathrm{K}_{\mathrm{co}}, \mathrm{T}_{\mathrm{io}}, \mathrm{T}_{\mathrm{do}}=\alpha\right.$ $\mathrm{T}_{\mathrm{io}}$ ) are calculated so that the new pseudo system has the specified relative damping $\left(\delta_{0}\right)$.

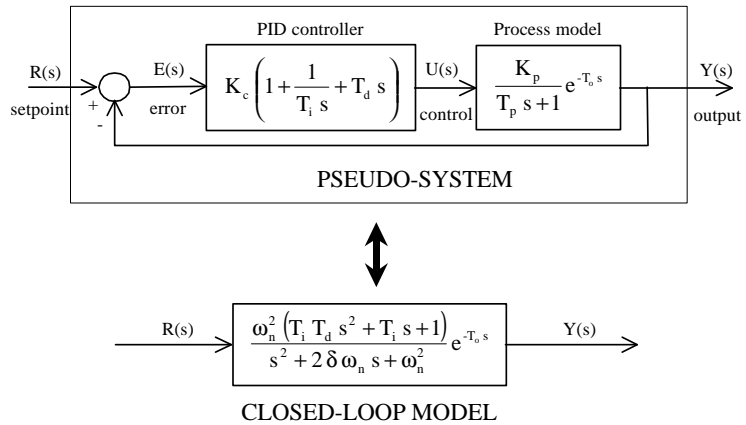

Fig. 2. Block diagrams of the pseudo-system and of the closed-loop model.

With this continuous approach (see Figure 2), the third (estimation of the process model) and the fourth stages (tuning of control parameters) are equivalent to two problems of pole placement. The first with a known controller and a process with known structure and unknown parameters. The second with a known process and a controller with known structure and unknown parameters. Thus the fact that the solution to these problems is purely analytical and appropriate for arriving at expressions of estimation and tuning useful in other strategies.

\subsection{Closed-loop response analysis.}

In the analysis stage the following four tasks are included (see the analysis section in Figure 3): filtering of the response in order to remove the effect of the controller's zeros, analysis of relative maxima and minima, determination of the nature of the response and measurement of dynamic characteristics. Two possible cases are considered; oscillatory response when it presents overshoot, and overdamped response in the opposite case. In the oscillatory case, the choice has been made to approximate the decay ratio b/a, the pseudoperiod of oscillation and the instant at $50 \%$. In the overdamped case, the choice has been to approximate the instant at $50 \%$, the instant at $73 \%$ and the characteristic area $\mathrm{A}_{\mathrm{o}}$ (the area enclosed by the step response and the final steady-state). These choices are extensively discussed by González (1994):

\subsection{Estimation of the closed-loop model.}

Since the PID controller guarantees null error in steady-state, the gain of the closed-loop model is always the unit. The remaining three parameters $(\delta$, $\omega_{n}, T_{o}$ ) should be such that a good approximation of certain characteristics of the transient response is obtained. Table 1 summarizes the set of functions which will be used, some of which are very well known, see (Aström, and Hägglund, 1995). Others, however, are contributions made by González (1994).

Table 1 Relationship between the step response characteristics of a second order system with dead time and its parameters.

\begin{tabular}{ccc}
\hline Characteristic & Parameter dependence & Formula \\
\hline $\begin{array}{c}\mathrm{b} / \mathrm{a} \\
\delta<1\end{array}$ & $\mathrm{e}^{-\frac{2 \delta \pi}{\sqrt{1-\delta^{2}}}}$ & $(1)$ \\
\hline $\begin{array}{c}\mathrm{T} \\
\delta<1\end{array}$ & $\frac{2 \pi}{\omega_{\mathrm{n}} \sqrt{1-\delta^{2}}}$ & $(2)$ \\
\hline $\begin{array}{c}\mathrm{T}_{50} \\
0<\delta<1\end{array}$ & $\mathrm{~T}_{50}=\mathrm{T}_{\mathrm{o}}+\frac{0.2596 \delta^{2}+0.3658 \delta+1.0479}{\omega_{\mathrm{n}}}$ & \\
\hline $\begin{array}{c}\mathrm{T}_{50} \\
1<\delta<2\end{array}$ & $\mathrm{~T}_{50}=\mathrm{T}_{\mathrm{o}}+\frac{1.1863 \delta+0.4426}{\omega_{\mathrm{n}}}$ \\
\hline $\begin{array}{c}\mathrm{T}_{73} \\
1<\delta<2\end{array}$ & $\mathrm{~T}_{73}=\mathrm{T}_{\mathrm{o}}+\frac{2.5966 \delta-0.0477}{\omega_{\mathrm{n}}}$ \\
\hline $\begin{array}{c}\mathrm{A}_{\mathrm{o}} \\
\delta>0\end{array}$ & $\mathrm{~A}_{\mathrm{o}}=\mathrm{T}_{\mathrm{o}}+\frac{2 \delta}{\omega_{\mathrm{n}}}$ \\
\hline
\end{tabular}

In the oscillatory case, the following sequence has been selected for the estimation of parameters (see the closed-loop estimation section in Figure 3): $\delta$ is obtained as a function of b/a, given by the inverse function formula (1) in table $1, \omega_{\mathrm{n}}$ is obtained as a function of $\delta$ and T, derived from formula (2) of table 1 , and $\mathrm{T}_{\mathrm{o}}$ is obtained as a function of $\delta, \omega_{\mathrm{n}}$ and $\mathrm{T}_{50}$, derived from formula (3) in table 1 . In contrast to the oscillatory case, the overdamped case does not have a characteristic unequivocally related with one of the parameters. This forces one to have to resolve a system of three transcendental equations with three unknowns, specifically the system formed by items (4), (5), and (6) in Table 1.

\subsection{Estimation of the process model.}

The equivalence between the transfer functions of the pseudo-system and of the closed-loop model has been made basing on the following hypotheses (see Figure 2):

a) The process model and the closed-loop model are continuous first and second order system, respectively, with dead time.

b) The controller is a continuous non-interactive PID. 


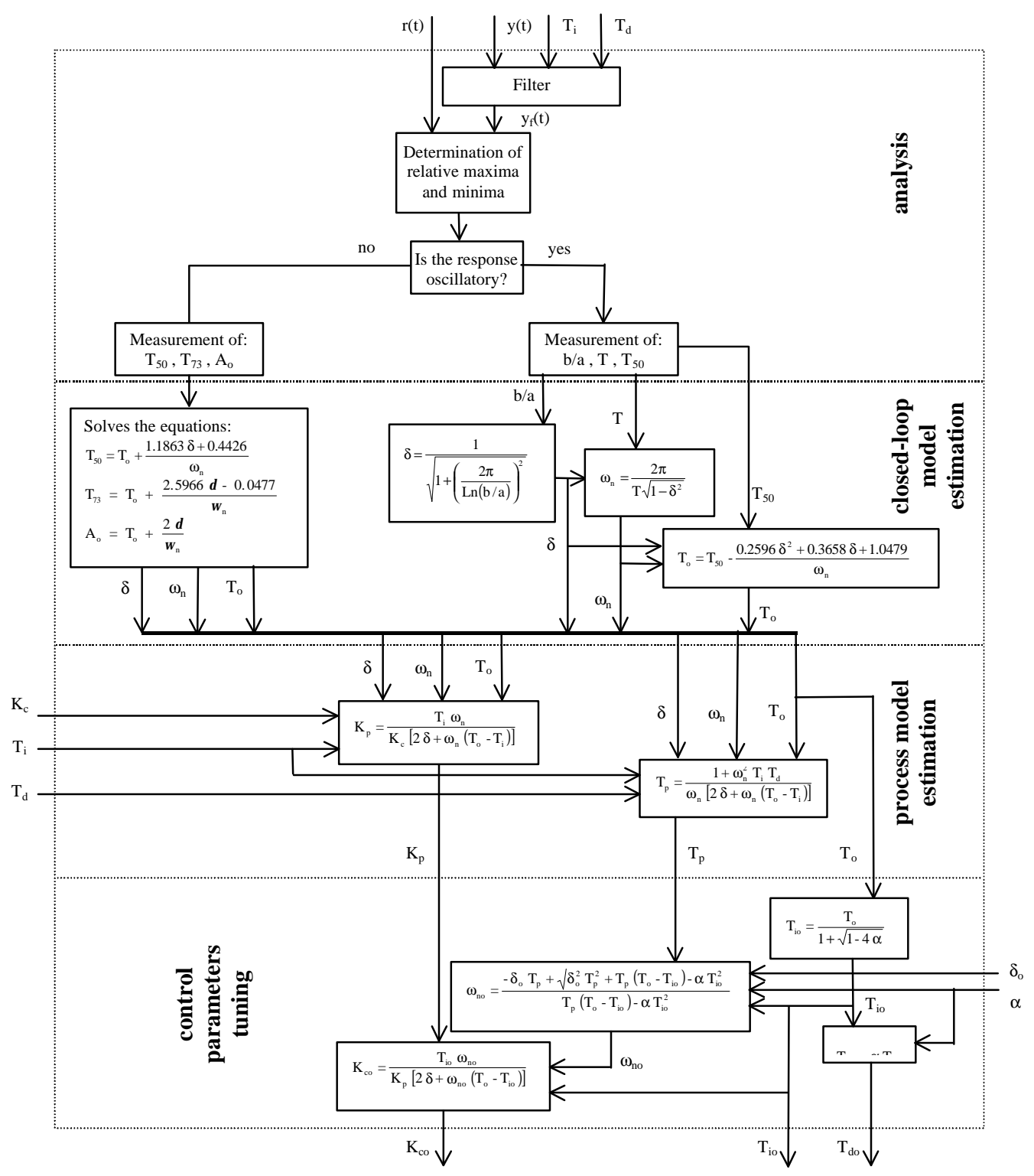

Fig. 3. The auto-tuning method in detail.

c) The apparent dead time of the process model is the same as the closed-loop model.

d) The zeros of the controller and of the process are transmitted at the system output.

With these hypotheses, if the control parameters and the parameters of the closed-loop model are known, it is possible to obtain the parameters of the process model using the following expressions (see the process estimation section in Figure 3):

$\mathrm{T}_{\mathrm{o}}$ equals the dead time of the closed-loop model

$$
\begin{aligned}
\mathrm{T}_{\mathrm{p}} & =\frac{1+\omega_{\mathrm{n}}^{2} \mathrm{~T}_{\mathrm{i}} \mathrm{T}_{\mathrm{d}}}{\omega_{\mathrm{n}}\left[2 \delta+\omega_{\mathrm{n}}\left(\mathrm{T}_{\mathrm{o}}-\mathrm{T}_{\mathrm{i}}\right)\right]} \\
\mathrm{K}_{\mathrm{p}} & =\frac{\mathrm{T}_{\mathrm{i}} \omega_{\mathrm{n}}}{\mathrm{K}_{\mathrm{c}}\left[2 \delta+\omega_{\mathrm{n}}\left(\mathrm{T}_{\mathrm{o}}-\mathrm{T}_{\mathrm{i}}\right)\right]}
\end{aligned}
$$

These expressions have been possible using Padé's first-order approximation for the dead time and assuming the associated pole in this approximation is cancelled by a zero controller, so that the following condition between $T_{i}, T_{d}$ and $T_{o}$ should be verified. with $0 \leq \mathrm{T}_{\mathrm{d}} / \mathrm{T}_{\mathrm{i}} \leq 0.25$.

$$
\mathrm{T}_{\mathrm{i}}=\frac{\mathrm{T}_{\mathrm{o}}}{1+\sqrt{1-4 \frac{\mathrm{T}_{\mathrm{d}}}{\mathrm{T}_{\mathrm{i}}}}}
$$

\subsection{Tuning of control parameters.}

The new control parameters, $T_{i o}, T_{d o}=\alpha T_{i o}$ and $K_{c o}$ will take the system to a new operating condition with other characteristics $\delta_{\mathrm{o}}$ and $\omega_{\mathrm{no}}$ but the same dead time $T_{0}$. In the new situation, the formulas (7) and (8) should also be verified along with condition (9). Assuming that the process model and therefore its parameters are the same as in the previous situation, the following tuning formulas are obtained:

$$
\begin{aligned}
\mathrm{T}_{\mathrm{io}} & =\frac{\mathrm{T}_{\mathrm{o}}}{1+\sqrt{1-4 \alpha}} \\
\mathrm{T}_{\mathrm{do}} & =\alpha \mathrm{T}_{\mathrm{io}} \\
\mathrm{K}_{\mathrm{co}} & =\frac{\mathrm{T}_{\mathrm{io}} \omega_{\mathrm{no}}}{\mathrm{K}_{\mathrm{p}}\left[2 \delta+\omega_{\mathrm{no}}\left(\mathrm{T}_{\mathrm{o}}-\mathrm{T}_{\mathrm{io}}\right)\right]}
\end{aligned}
$$


Where it is observed that: $\mathrm{T}_{\mathrm{io}}$ is independent of specification $\delta_{0}, T_{\mathrm{do}}$ maintains the specified ratio $\alpha$ with $\mathrm{T}_{\mathrm{io}}$, and $\mathrm{K}_{\mathrm{co}}$ is conditioned by specification $\delta_{\mathrm{o}}$ through $\omega_{\text {no }}$ (see the control parameters tuning section in Figure 3). Concretely, having specified the damping, the natural frequency of the closed-loop model is forced to take on the following value:

$$
\omega_{\mathrm{no}}=\frac{-\delta_{\mathrm{o}} \mathrm{T}_{\mathrm{p}}+\sqrt{\delta_{\mathrm{o}}^{2} \mathrm{~T}_{\mathrm{p}}^{2}+\mathrm{T}_{\mathrm{p}}\left(\mathrm{T}_{\mathrm{o}}-\mathrm{T}_{\mathrm{io}}\right)-\alpha \mathrm{T}_{\mathrm{io}}^{2}}}{\mathrm{~T}_{\mathrm{p}}\left(\mathrm{T}_{\mathrm{o}}-\mathrm{T}_{\mathrm{io}}\right)-\alpha \mathrm{T}_{\mathrm{io}}^{2}}
$$

to maintain the same $T_{p}$ of the process model.

\section{VALIDATION OF THE METHOD}

To evaluate the proposed auto-tuning method, simulations have been carried out using a lot of continuous processes encompassing the characteristics of the most of industrial processes, from processes with dominant time constant, to processes with dominant dead time. From among the numerous tests realized, see (González, 1994), the most noteworthy are the tests of convergence, and the tests of specification's tracking. The convergence tests have as objective to verify if after a change in the dynamic of the process, which caused the system to move away from the temporal response specification, the auto-tuning method was to be able to adapt the control parameters to the new situation in the possible shortest time (least number of cycles). The second tests have as objective to verify if after a change in the temporal response specification, the auto-tuning method was to be able to adapt the control parameters to the new situation in the possible shortest time (least number of cycles).

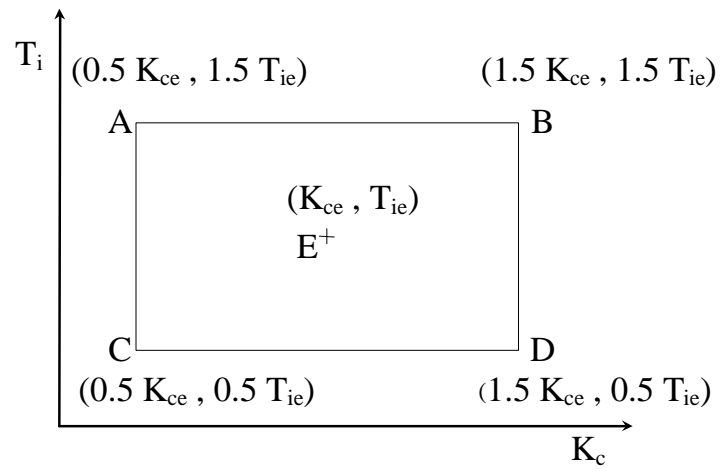

Fig. 4. Relative points used in convergence tests to simulate dynamic changes in the process.

The tests of convergence have been carried out simulating the change in the process dynamic as a change in the control parameters. The justification is as follows: let be a process which has managed to be taken to a fixed specification $\left(\delta_{0}\right)$. The current set of parameters is indicated as point $\mathrm{E}$ on the parametric control plane (plane $\mathrm{K}_{\mathrm{c}}, \mathrm{T}_{\mathrm{i}}$ ). Let $\mathrm{A}, \mathrm{B}, \mathrm{C}$ and $\mathrm{D}$ be the vertices of the rectangle represented in Figure 4, which have been calculated from point $E$ as combinations of a $50 \%$ increase or decrease in the control parameters. Simulating the step from point A to point $\mathrm{E}$, assuming that in both points the specification $\left(\delta_{0}\right)$ is fulfilled, is equivalent to simulating the step from the corresponding point $\mathrm{A}^{\prime}$ on the parametric plane of the process to point $\mathrm{E}^{\prime}$, and it is therefore equivalent to simulating a dynamic change in the process. Analogous interpretations have the steps from $\mathrm{B}$ to $\mathrm{E}$, from $\mathrm{C}$ to $\mathrm{E}$ and from $\mathrm{D}$ to $\mathrm{E}$.

\section{APPLICATIONS OF THE METHOD}

The system of Figure 5 has been developed to test in simulation and real time the auto-tuning method. The system consists of four functional blocks, which are briefly described next, and several auxiliary blocks to display the evolution of the control parameters, the control signal and the process output.

- The "process" block represents the block of the same name in Figure 1. It could represent a simulated or real process. In the first case it is implemented with basic blocks of simulink (linear or non-linear), in the second case is a real plant connected to the computer through blocks of the "Real Time Toolbox" of Humusoft.

- The "PID" block represents the "controller" of the Figure 1. The control parameters $\left(K_{c}, T_{i} y\right.$ $\mathrm{T}_{\mathrm{d}}$ ) are input signals to the block, because they can be modified. The controller will be a continuous block, unless when the control was on a real process, because in this case a digital controller is necessary.

- The "autotuning" block includes the four blocks of Figure 1 (closed-loop response analyser, closed-loop model estimator, process model estimator and control parameters tuner).

- The "monitor" block, which is not present in Figure 1, is the characteristic block of the implementation in SIMULINK. It is in charge of coordinating the others blocks of the system in order to get successive tuning for the control parameters.

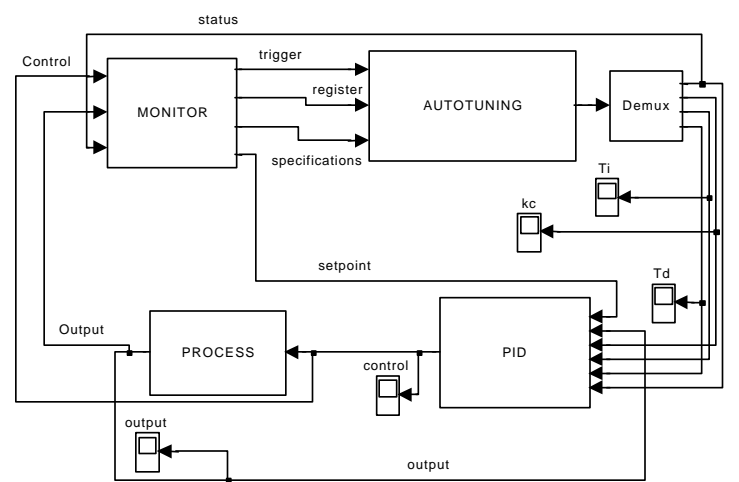

Fig. 5. Diagram of the method in SIMULINK

In the diagram of Figure 5 coexist two loops, the typical control closed-loop and the tuning loop The "monitor" block is in charge of introducing sudden changes in the setpoint, while the other signals will be consequence of these changes:

- The trigger signal, initially to zero, showing the inactivity of the "autotuning" block. When this signal changes to one, it enables the calculus of the new control parameters. 
- The register signal, that is a vector generated by the "monitor" with the evolution of the setpoint, the control signal and the output process during a certain interval. The register will have in any time the adequated size for that the "autotuning" block can analyse the closed-loop response to the setpoint change.

- The specifications composed of the set $\left(\delta_{\mathrm{o}} \mathrm{y} \alpha\right)$. Where $\delta_{0}$ represents the specified damping for the closed-loop response and $\alpha$ represents the constrain for the ratio between the derivative and integral time constants of the controller.

- The status signal, it is generated by the "autotuning" block to show the end of the calculus of the new control parameters. The signal is important part in the generation of the trigger signal and also it is used to communicate to the controller that it must update their control parameters.

- The current control parameters $\left(\mathrm{K}_{\mathrm{c}}, \mathrm{T}_{\mathrm{i}} \mathrm{y} \mathrm{T}_{\mathrm{d}}\right)$.

The method has been successfully tested with a big number of processes considered in the bibliography on PID controllers. As more significant example of this, the application to a process with multiple equal poles is presented. It has been used by controller manufactures as test cases for a long time (see Aström, and Hägglund 1999). The transfer function of this process is

$$
\frac{1}{(\mathrm{~s}+1)^{\mathrm{n}}} \mathrm{n}=1,2,3,4, \ldots, 8
$$

Since the auto-tuning proposed needs first order model with dead time and only can be applied if the dead time is not equal to zero, there is to discard the case $n=1$ and for the other cases $n=2, \ldots, 8$ is necessary to have an initial model with these characteristics. The Table 2 shows the time constant and the dead time of the models estimated in openloop using the moments method (Aström and Hägglund, 1995), the gain is not included because its value is one in every cases. As significant characteristic of the estimated parameters notice that: $\mathrm{T}_{\mathrm{p}}+\mathrm{T}_{\mathrm{o}}=\mathrm{n}$. But while $\mathrm{T}_{\mathrm{p}}$ doesn't experiment a great change from $n=2$ to $n=8$, whether experiment it the dead time, therefore the more large it is $n$ more dominant is the dead time over the time constant.

$\underline{\text { Table } 2 \text { Time constants and dead times estimated in }}$ open-loop for the system described by (12).

\begin{tabular}{ccc}
\hline $\mathrm{n}$ & $\mathrm{T}_{\mathrm{p}}$ & $\mathrm{T}_{\mathrm{o}}$ \\
\hline 2 & 1.41 & 0.59 \\
3 & 1.73 & 1.27 \\
4 & 2.00 & 2.00 \\
5 & 2.24 & 2.76 \\
6 & 2.45 & 3.55 \\
7 & 2.64 & 4.36 \\
8 & 2.80 & 5.20 \\
\hline
\end{tabular}

Since $T_{i}$ will be equal to $T_{o} / 2$ for the PI controller and will be function of $T_{0}$ and $\alpha$ for the PID controller. Starting from the third column of Table 2, the $T_{i}$ that the method will assign initially to each case is immediately known. On the other hand the proportional gain depends also of the specification $\delta_{0}$. But these parameters are not always good to achieve the desired specification, Table 3 shows the relative deviations $\left(100 \mid \delta_{\mathrm{m}}-\delta_{\mathrm{o}} / \delta_{\mathrm{o}}\right)$ in percentage between the specified $\left(\delta_{\mathrm{o}}\right)$ and the measured $\left(\delta_{\mathrm{m}}\right)$ damping for PID control with $\alpha=0.1$ and four values $(0.2,0.3,0.4$ and $0.5)$ of $\delta_{0}$. Notice that, as was foreseeable, the more large it is $\mathrm{n}$ more large is the relative deviation, since increase the difficulty to controlling the process. However with five iterations of the auto-tuning method the control parameters converge to other values, and the damping of the closed-loop response is more near the specified value $\delta_{0}$, (see quantities in italic and bold type of Table 3 ). On the whole the relative deviations are least than $5 \%$, which is an indicative of the method's goodness. There is another cases in which the method have been unable to get relative deviations least than $10 \%$, but the transient responses are admissibles due to the difficulty to controlling the processes. Similar results has been obtained with PI control.

Table 3 Relative deviations for the process (12), with PID controller with $\alpha=0.1$, in the initial conditions (quantities in normal type) and after five autotuning (quantities in italic and bold type)

\begin{tabular}{|c|c|c|c|c|c|c|c|c|c|}
\hline & \multicolumn{9}{|c|}{$\delta_{o}$} \\
\hline & \multicolumn{3}{|c|}{0.2} & \multicolumn{2}{|c|}{0.3} & \multicolumn{2}{|c|}{0.4} & \multicolumn{2}{|c|}{0.5} \\
\hline & 2 & 28.0 & 1.40 & 22.0 & 0.90 & 16.0 & 0.40 & 12 & 0.08 \\
\hline & 3 & 40.0 & 1.90 & 28.0 & 1.10 & 21.0 & 0.30 & 14.0 & 0.20 \\
\hline & 4 & 53.0 & 3.30 & 36.0 & 2.00 & 25.0 & 1.00 & 18.0 & 0.80 \\
\hline $\mathrm{N}$ & 5 & 64.0 & 4.00 & 43.0 & 3.00 & 31.0 & 4.00 & 22.0 & 4.40 \\
\hline & 6 & 74.0 & 4.00 & 51.0 & 7.00 & 36.0 & 1.00 & 26 & 8.20 \\
\hline & 7 & 83.0 & 2.20 & 57.0 & 3.30 & 42.0 & 6.00 & 31 & 14.2 \\
\hline & 8 & 76.0 & 13.0 & 54.0 & 6.00 & 40.0 & 14.0 & 31 & 31 \\
\hline
\end{tabular}
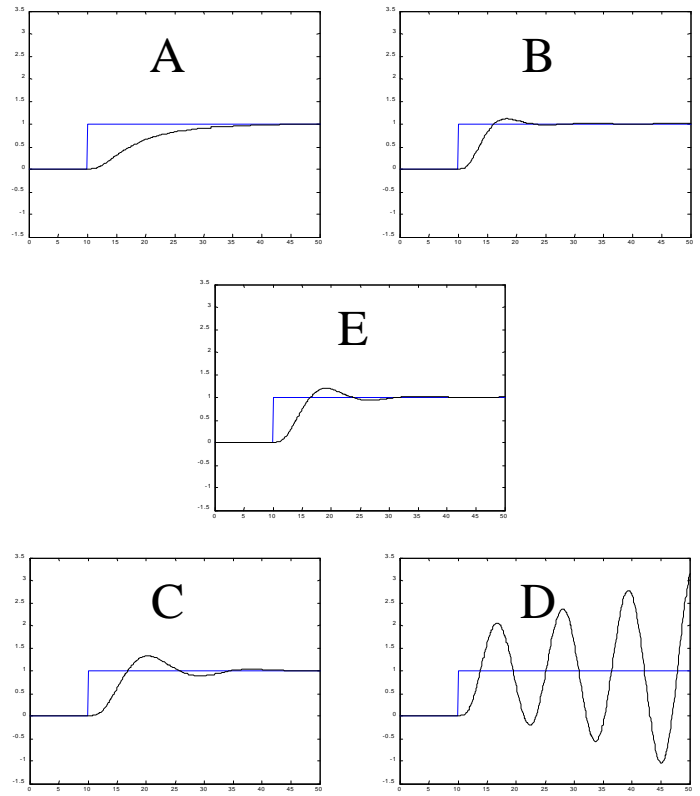

Fig. 6. Typical closed-loop responses in the five situations (A, B, C, D and E) of convergence test. 
Figure 6 shows the closed-loop responses in the four departures points (A, B, C and D) and in the final point $(\mathrm{E})$ for the convergence test when the process (12) with $n=4$, the PID controller with $\alpha=0.1$, and the specification $\delta_{0}=0.4$ are selected. While the responses in points $\mathrm{B}$ and $\mathrm{C}$ have a damping near to the specified, the response in point $\mathrm{A}$ is very damped and the response in point $\mathrm{D}$ is unstable. This is typical in all the convergence tests. Figure 7 shows the results on the parametric control plane, the points of a trayectory are marked with the same symbol and they are joined with a line. Notice that: 1) in the four cases the control parameters finish in the point E , 2) on the whole the points of these trayectories are placed near the line that joint $\mathrm{B}$ and $\mathrm{C}$, because in these positions the desired specification is achieved, 3) the specification re-establishes itself in the first or second tuning. Therefore Figure 7 is an indicative of the method's goodness. Similar results has been obtained with PI control, others specifications, and value of $n$.

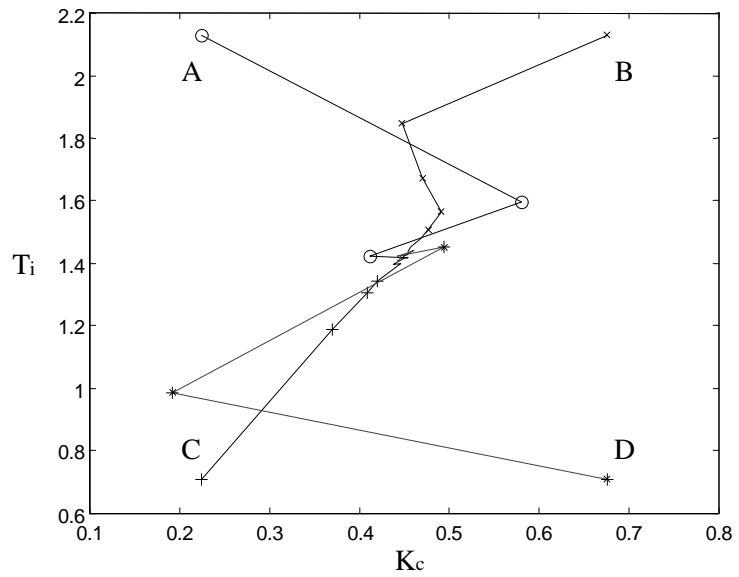

Fig. 7. Typical trayectories on the $\left(\mathrm{K}_{\mathrm{c}}, \mathrm{T}_{\mathrm{i}}\right)$ plane in the convergence test.
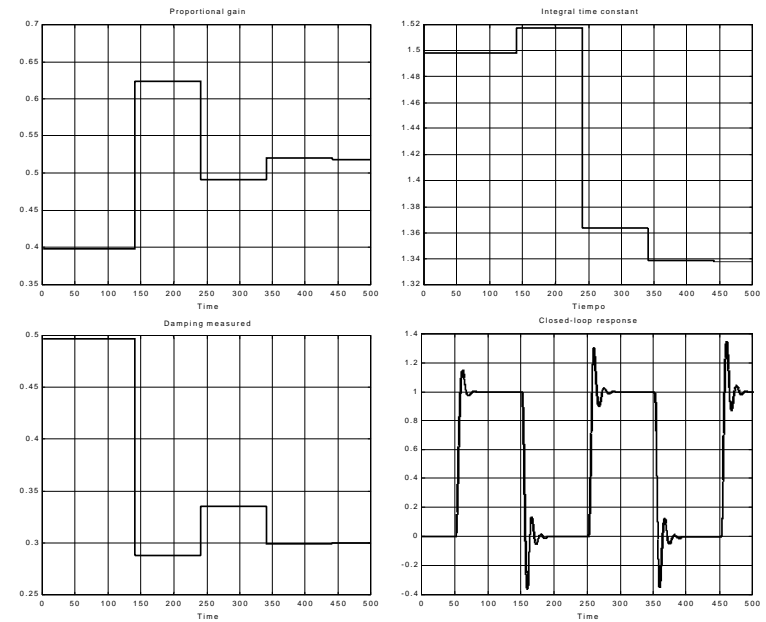

Fig. 8. Typical results when the specification changes

Figure 8 is an example of what happen if the specification $\left(\delta_{0}\right)$ change. The process (12) with $\mathrm{n}=4$, the PID controller with $\alpha=0.1$, and the specification change from $\delta_{0}=0.5$ to $\delta_{0}=0.3$ have been selected. Notice that the convergence in the control parameters have been achieved in three tuning, but the damping of the closed-loop response is very near of the new specification (0.3) since the first tuning. The Figure 8 is an indicative of the method's goodness to track a variable damping making use of the setpoint changes.

\section{CONCLUSIONS}

The numerous tests carried out in simulation and in real time lead to the following conclusions:

a) In all the situations of underdamped response, the auto-tuning method proposed has managed to re-establish the desired response characteristic within the first or second cycle.

b) In the cases of unstable or overdamped response, the method has proven itself efficient in bringing the system out of such a situation and taking it to a new, more favourable one within a single cycle.

c) The method proposed is applicable to a wide spectrum of industrial processes with a monotone step response in open loop. It produces the best performance with respect to other methods for process with dominant dead time, which are considered the most difficult ones to control.

\section{ACKNOWLEDGEMENTS}

The authors wish to acknowledge the economical support of the Spanish CICYT (Comisión Interministerial de Ciencia y Tecnología), under Grant TAP 96-0404.

\section{REFERENCES}

Aström, K.J., T. Hägglund C.C. Hang and W. K. Ho (1993). Automatic tuning and adaptation for PID controllers - a survey. Control Eng. Practice, Vol 1, No 4, 699-714.

Aström, K.J. and T. Hägglund (1995). PID Controllers: Theory, Design, and Tuning $\left(2^{\text {nd }}\right.$ Edition). Research Triangle Park, NC: Instrument Society of America.

Aström, K.J. and T. Hägglund (1999). Benchmark Systems for PID Control. PID'00, IFAC Workshop on Digital Control, Past, Present and Future of PID control.

González, A. M. (1994). Un planteamiento continuo de la autosintonía de controladores PI y PID. $\mathrm{PhD}$ dissertation, Dpto. de Informática $\mathrm{y}$ Automática, UNED.

Kraus, T.W. and T.J. Myron (1984). Self-tuning PID controller uses pattern recognition approach. Control Engineering, June, 106-111.

Morilla, F. (1987). Contribución a los métodos de autosintonía de reguladores PID. $\mathrm{PhD}$ dissertation, Dpto. de Informática y Automática, UNED.

Seem, J.E. (1996). A new pattern recognition adaptive controller. $13^{\text {th }}$ IFAC World Congress, K, 121-126. 